Commun. Korean Math. Soc. 28 (2013), No. 2, pp. 335-351

http://dx.doi.org/10.4134/CKMS.2013.28.2.335

\title{
A HYBRID METHOD FOR A COUNTABLE FAMILY OF LIPSCHITZ GENERALIZED ASYMPTOTICALLY QUASI-NONEXPANSIVE MAPPINGS AND AN EQUILIBRIUM PROBLEM
}

Prasit Cholamjiak, Watcharaporn Cholamjiak, and Suthep Suantai

\begin{abstract}
In this paper, we introduce a new iterative scheme for finding a common element of the fixed points set of a countable family of uniformly Lipschitzian generalized asymptotically quasi-nonexpansive mappings and the solutions set of equilibrium problems. Some strong convergence theorems of the proposed iterative scheme are established by using the concept of $W$-mappings of a countable family of uniformly Lipschitzian generalized asymptotically quasi-nonexpansive mappings.
\end{abstract}

\section{Introduction}

Let $C$ be a nonempty, closed and convex subset of a real Banach space $E$ and let $T: C \rightarrow C$. We denote by $F(T)$ the set of fixed points of $T$, that is, $F(T)=\{x \in C: x=T x\}$. Then $T$ is said to be

(i) nonexpansive if $\|T x-T y\| \leq\|x-y\|$ for all $x, y \in C$;

(ii) asymptotically nonexpansive if there exists a sequence $k_{n} \geq 1, \lim _{n \rightarrow \infty} k_{n}$ $=1$ and

for all $x, y \in C$ and $n \in \mathbb{N}$;

$$
\left\|T^{n} x-T^{n} y\right\| \leq k_{n}\|x-y\|
$$

(iii) asymptotically quasi-nonexpansive if there exists a sequence $k_{n} \geq 1$, $\lim _{n \rightarrow \infty} k_{n}=1$ and

$$
\left\|T^{n} x-p\right\| \leq k_{n}\|x-p\|
$$

for all $x \in C, p \in F(T)$ and $n \in \mathbb{N}$;

(iv) generalized asymptotically nonexpansive [24] if there exist nonnegative real sequences $\left\{k_{n}\right\}$ and $\left\{c_{n}\right\}$ with $k_{n} \geq 1, \lim _{n \rightarrow \infty} k_{n}=1$ and $\lim _{n \rightarrow \infty} c_{n}=0$ such that

$$
\left\|T^{n} x-T^{n} y\right\| \leq k_{n}\|x-y\|+c_{n}
$$

for all $x, y \in C$ and $n \in \mathbb{N}$;

Received May 8, 2012

2010 Mathematics Subject Classification. 47H09, 47H10.

Key words and phrases. generalized asymptotically quasi-nonexpansive mapping, hybrid method, common fixed point, strong convergence, $W$-mapping. 
(v) generalized asymptotically quasi-nonexpansive [24] if there exist nonnegative real sequences $\left\{k_{n}\right\}$ and $\left\{c_{n}\right\}$ with $k_{n} \geq 1, \lim _{n \rightarrow \infty} k_{n}=1$ and $\lim _{n \rightarrow \infty} c_{n}=0$ such that

$$
\left\|T^{n} x-p\right\| \leq k_{n}\|x-p\|+c_{n}
$$

for all $x \in C, p \in F(T)$ and $n \in \mathbb{N}$;

(vi) uniformly $L$-Lipschitzian if there exists a constant $L>0$ such that

$$
\left\|T^{n} x-T^{n} y\right\| \leq L\|x-y\|
$$

for all $x, y \in C$ and $n \in \mathbb{N}$.

It is clear that a class of generalized asymptotically quasi-nonexpansive mappings includes as special cases the class of generalized asymptotically nonexpansive mappings, the class of asymptotically nonexpansive mappings, and the class of nonexpansive mappings. However, the converse of each of above statements may be not true (see [24]).

In 1953, Mann [13] introduced the following iterative procedure to approximate a fixed point of a nonexpansive mapping $T$ in a Hilbert space $H$ :

$$
x_{n+1}=\alpha_{n} x_{n}+\left(1-\alpha_{n}\right) T x_{n}, \quad \forall n \in \mathbb{N},
$$

where the initial point $x_{1}$ is taken in $C$ arbitrarily and $\left\{\alpha_{n}\right\}$ is a sequence in $[0,1]$.

However, we note that Mann's iteration process (1.1) has only weak convergence, in general; for instance, see [1, 10, 22].

Question: How can we modify Mann's iteration process to obtain strong convergence theorems?

In 2003, Nakajo and Takahashi [18] introduced a modification of Mann iteration which is called $C Q$ method for a single nonexpansive mapping $T$ in a Hilbert space. They proved the following theorem:

Theorem 1.1. Let $C$ be a closed convex subset of a Hilbert space $H$ and let $T: C \rightarrow C$ be a nonexpansive mapping such that $F(T) \neq \emptyset$. Assume that $\left\{\alpha_{n}\right\}_{n=0}^{\infty}$ is a sequence in $[0,1]$ such that $\alpha_{n} \leq 1-\delta$ for some $\delta \in(0,1]$. Define a sequence $\left\{x_{n}\right\}_{n=0}^{\infty}$ in $C$ by the following manner:

$$
\left\{\begin{array}{l}
x_{0} \in C \text { chosen arbitrarily, } \\
y_{n}=\alpha_{n} x_{n}+\left(1-\alpha_{n}\right) T x_{n}, \\
C_{n}=\left\{z \in C:\left\|y_{n}-z\right\| \leq\left\|x_{n}-z\right\|\right\}, \\
Q_{n}=\left\{z \in C:\left\langle x_{0}-x_{n}, x_{n}-z\right\rangle \geq 0\right\}, \\
x_{n+1}=P_{C_{n} \cap Q_{n}} x_{0} .
\end{array}\right.
$$

Then $\left\{x_{n}\right\}$ defined by (1.2) converges strongly to $P_{F(T)} x_{0}$.

In 2006, Kim and Xu [12] extended the result of Nakajo and Takahashi [18] from a single nonexpansive mapping to a single asymptotically nonexpansive mapping in a Hilbert space. They proved the following theorem: 
Theorem 1.2. Let $C$ be a nonempty bounded closed convex subset of a Hilbert space $H$ and let $T: C \rightarrow C$ be an asymptotically nonexpansive mapping with a sequence $\left\{k_{n}\right\}$ such that $k_{n} \rightarrow 1$ as $n \rightarrow \infty$. Assume that $\left\{\alpha_{n}\right\}_{n=0}^{\infty}$ is a sequence in $[0,1]$ such that $\lim _{\sup _{n \rightarrow \infty}} \alpha_{n}<1$. Define a sequence $\left\{x_{n}\right\}$ in $C$ by the following manner:

$$
\left\{\begin{array}{l}
x_{0} \in C \text { chosen arbitrarily, } \\
y_{n}=\alpha_{n} x_{n}+\left(1-\alpha_{n}\right) T^{n} x_{n}, \\
C_{n}=\left\{z \in C:\left\|y_{n}-z\right\|^{2} \leq\left\|x_{n}-z\right\|^{2}+\theta_{n}\right\} \\
Q_{n}=\left\{z \in C:\left\langle x_{0}-x_{n}, x_{n}-z\right\rangle \geq 0\right\} \\
x_{n+1}=P_{C_{n} \cap Q_{n}} x_{0},
\end{array}\right.
$$

where $\theta_{n}=\left(1-\alpha_{n}\right)\left(k_{n}^{2}-1\right)(\operatorname{diam} C)^{2} \rightarrow 0$. Then $\left\{x_{n}\right\}$ defined by $(1.3)$ converges strongly to $P_{F(T)} x_{0}$.

In 2008, Takahashi et al. [28] proved the following theorem by using a hybrid method which is different from Nakajo and Takahashi's hybrid method. Such a method is called the shrinking projection method.

Theorem 1.3 ([28]). Let $H$ be a Hilbert space and let $C$ be a nonempty closed convex subset of $H$. Let $T$ be a nonexpansive mapping of $C$ into $H$ such that $F(T) \neq \emptyset$ and let $x_{0} \in H$. For $C_{1}=C$ and $u_{1}=P_{C_{1}} x_{0}$, define a sequence $\left\{u_{n}\right\}$ of $C$ as follows:

$$
\left\{\begin{array}{l}
y_{n}=\alpha_{n} u_{n}+\left(1-\alpha_{n}\right) T u_{n} \\
C_{n+1}=\left\{z \in C_{n}:\left\|y_{n}-z\right\| \leq\left\|u_{n}-z\right\|\right\} \\
u_{n+1}=P_{C_{n+1}} x_{0}, \quad n \in \mathbb{N}
\end{array}\right.
$$

where $0 \leq \alpha_{n} \leq a<1$ for all $n \in \mathbb{N}$. Then $\left\{u_{n}\right\}$ converges strongly to $z_{0}=P_{F(T)} x_{0}$.

Since 2003, to obtain strong convergence of nonlinear mappings, the CQ method and the shrinking projection method have been studied by many authors (see $[12,14,15,16,17,18,19,20,23,25,26,29,31,32]$ ).

Let $F: C \times C \rightarrow \mathbb{R}$ be a bifunction. The equilibrium problem for a bifunction $F$ is to find a point $x^{*} \in C$ such that

$$
F\left(x^{*}, y\right) \geq 0, \quad \forall y \in C .
$$

We denote the solutions set of (1.5) by $\operatorname{EP}(F)$.

Question. How can we construct iteration process to obtain strong convergence theorems for finding a common element of the solutions set of an equilibrium problem and the fixed points set?

Recently, Tada and Takahashi [26] proposed a new iteration for finding a common element of the solutions set of an equilibrium problem and the fixed points set of a nonexpansive mapping $T$ in a Hilbert space $H$ and then proved the following theorem: 
Theorem 1.4 ([26]). Let $H$ be a Hilbert space and $C$ a closed convex subset of $H$. Let $F: C \times C \rightarrow R$ be a bifunction and $T: C \rightarrow C$ a nonexpansive mapping such that $F(T) \cap E P(F) \neq \emptyset$. For an initial point $x_{1}=x \in C$, let a sequence $\left\{x_{n}\right\}$ be generated by

$$
\left\{\begin{array}{l}
F\left(u_{n}, y\right)+\frac{1}{r_{n}}\left\langle y-u_{n}, u_{n}-x_{n}\right\rangle \geq 0 \quad \forall y \in C, \\
y_{n}=\alpha_{n} x_{n}+\left(1-\alpha_{n}\right) T u_{n}, \\
C_{n}=\left\{z \in C:\left\|y_{n}-z\right\| \leq\left\|x_{n}-z\right\|\right\} \\
Q_{n}=\left\{z \in C:\left\langle x_{n}-z, x_{n}-x\right\rangle \leq 0\right\} \\
x_{n+1}=P_{C_{n} \cap Q_{n}} x, \quad \forall n \in \mathbb{N},
\end{array}\right.
$$

where $0 \leq \alpha_{n} \leq a<1$ and $\liminf _{n \rightarrow \infty} r_{n}>0$. Then $\left\{x_{n}\right\}$ converges strongly to $P_{F(T) \cap E P(F)} x$.

In the recent years, the problem of finding a common element of the solutions set of equilibrium problems and the fixed points set in the framework of Hilbert spaces and Banach spaces has been intensively studied by many authors (see, for example, $[2,3,5,30,4,6,7,9,11,21,27]$ and the references cited therein).

Let $\left\{T_{n}\right\}$ be a family of asymptotically nonexpansive mappings of $C$ into itself and let $\alpha_{i, n} \in[0,1]$ for all $i=1,2, \ldots, n$ and $n \in \mathbb{N}$.

In 2008, Nakajo et al. [17] introduced a new concept of the $W$-mapping as follows:

$$
\left\{\begin{array}{l}
U_{n, n}=\alpha_{n, n} T_{n}^{n}+\left(1-\alpha_{n, n}\right) I \\
U_{n-1, n}=\alpha_{n-1, n} T_{n-1}^{n} U_{n, n}+\left(1-\alpha_{n-1, n}\right) I \\
\vdots \\
U_{k, n}=\alpha_{k, n} T_{k}^{n} U_{k+1, n}+\left(1-\alpha_{k, n}\right) I \\
\vdots \\
W_{n}=U_{1, n}=\alpha_{1, n} T_{1}^{n} U_{2, n}+\left(1-\alpha_{1, n}\right) I .
\end{array}\right.
$$

Such a mapping $W$ is called the $W$-mapping generated by $T_{n}, \ldots, T_{2}, T_{1}$ and $\alpha_{n, n}, \ldots, \alpha_{2, n}, \alpha_{1, n}$.

Motivated by Nakajo et al. [17], we introduce a new approximation scheme for finding a common element of the fixed points set of a countable family of Lipschitz generalized asymptotically quasi-nonexpansive mappings and the solutions set of an equilibrium problem. Using a shrinking projection technique, strong convergence theorems are also established.

\section{Preliminaries}

In this section, we give some useful lemmas and definitions for proving our main theorem.

Let $H$ be a real Hilbert space with inner product $\langle\cdot, \cdot\rangle$ and norm $\|\cdot\|$ and $C$ a closed and convex subset of $H$. For every point $x \in H$, there exists a unique nearest point in $C$, denoted by $P_{C} x$, such that

$$
\left\|x-P_{C} x\right\| \leq\|x-y\|, \quad \forall y \in C .
$$


$P_{C}$ is called the metric projection of $H$ onto $C$. We know that $P_{C}$ is a nonexpansive mapping of $H$ onto $C$.

Lemma 2.1 ([14]). Let $C$ be a closed and convex subset of a real Hilbert space $H$ and $P_{C}$ the metric projection from $H$ onto $C$. Given $x \in H$ and $z \in C$. Then $z=P_{C} x$ if and only if $\langle x-z, y-z\rangle \leq 0$ for all $y \in C$.

Lemma 2.2 ([18]). Let $C$ be a nonempty, closed and convex subset of a real Hilbert space $H$ and $P_{C}: H \rightarrow C$ the metric projection from $H$ onto $C$. Then the following holds:

$$
\left\|y-P_{C} x\right\|^{2}+\left\|x-P_{C} x\right\|^{2} \leq\|x-y\|^{2}, \quad \forall x \in H, \forall y \in C .
$$

Lemma 2.3 ([14]). Let $H$ be a real Hilbert space. Then the followings hold:

(i) $\|x-y\|^{2}=\|x\|^{2}-\|y\|^{2}-2\langle x-y, y\rangle, \forall x, y \in H$;

(ii) $\|t x+(1-t) y\|^{2}=t\|x\|^{2}+(1-t)\|y\|^{2}-t(1-t)\|x-y\|^{2}, \forall t \in[0,1]$ and $x, y \in H$.

Lemma 2.4 ([25]). Let $C$ be a nonempty, closed and convex subset of a real Hilbert space $H$. Given $x, y, z \in H$ and also given $a \in \mathbb{R}$, the set

$$
\left\{v \in C:\|y-v\|^{2} \leq\|x-v\|^{2}+\langle z, v\rangle+a\right\}
$$

is convex and closed.

For solving the equilibrium problem, let us give the following assumptions for $F$ and the set $C$ :

(A1) $F(x, x)=0$ for all $x \in C$;

(A2) $F$ is monotone, i.e., $F(x, y)+F(y, x) \leq 0$ for all $x, y \in C$;

(A3) for each $x, y, z \in C$, $\lim \sup _{t \downarrow 0} F(t z+(1-t) x, y) \leq F(x, y)$;

(A4) $F(x, \cdot)$ is convex and lower semicontinuous for each $x \in C$.

Lemma $2.5([2])$. Let $C$ be a nonempty, closed and convex subset of a real Hilbert space $H$. Let $F$ be a bifunction from $C \times C$ to $\mathbb{R}$ satisfying (A1)-(A4) and let $r>0$ and $x \in H$. Then, there exists $z \in C$ such that

$$
F(x, y)+\frac{1}{r}\langle y-z, z-x\rangle \geq 0, \quad \forall y \in C .
$$

Lemma 2.6 ([9]). For $r>0, x \in H$, defined the mapping $T_{r}: H \rightarrow C$ as follows:

$$
T_{r}(x)=\left\{z \in C: F(z, y)+\frac{1}{r}\langle y-z, z-x\rangle \geq 0, \quad \forall y \in C\right\} .
$$

Then the followings hold:

(1) $T_{r}$ is single-value;

(2) $T_{r}$ is firmly nonexpansive, i.e., for any $x, y \in H$,

$$
\left\|T_{r} x-T_{r} y\right\|^{2} \leq\left\langle T_{r} x-T_{r} y, x-y\right\rangle ;
$$

(3) $F\left(T_{r}\right)=E P(F)$;

(4) $\operatorname{EP}(F)$ is closed and convex. 
Lemma 2.7 ([29]). Let $p>1, r>0$ be two fixed numbers. Then a Banach space $E$ is uniformly convex if and only if there exists a continuous, strictly increasing, and convex function $g:[0, \infty) \rightarrow[0, \infty)$ with $g(0)=0$ such that

$$
\|\lambda x+(1-\lambda) y\|^{p} \leq \lambda\|x\|^{p}+(1-\lambda)\|y\|^{p}-\omega_{p}(\lambda) g(\|x-y\|)
$$

for all $x, y \in B_{r}(0)=\{x \in E:\|x\| \leq r\}$ and $\lambda \in[0,1]$ where $\omega_{p}(\lambda)=$ $\lambda(1-\lambda)^{p}+\lambda^{p}(1-\lambda)$.

Lemma 2.8 ([8]). Let $C$ be a nonempty subset of a Banach space $E$ and $T: C \rightarrow C$ be a uniformly L-Lipschitzian. Let $\left\{x_{n}\right\}$ be a sequence in $C$ such that $\lim _{n \rightarrow \infty}\left\|x_{n+1}-x_{n}\right\|=0$ and $\lim _{n \rightarrow \infty}\left\|x_{n}-T^{n} x_{n}\right\|=0$. Then $\lim _{n \rightarrow \infty}\left\|x_{n}-T x_{n}\right\|=0$.

Lemma 2.9 ([8]). Let $C$ be a closed and convex subset of a real Hilbert space $H$. Let $T: C \rightarrow C$ be a uniformly L-Lipschitzian and generalized asymptotically quasi-nonexpansive mapping such that $F(T) \neq \emptyset$. Then $F(T)$ is a closed and convex subset of $C$.

\section{Results for families of mappings}

In this section, we prove some lemmas concerning the $W$-mapping in a real Banach space.

Lemma 3.1. Let $C$ be a nonempty convex subset of a real Banach space E. Let $\left\{T_{i}\right\}_{i=1}^{\infty}$ be a uniformly $L_{i}$-Lipschitzian and generalized asymptotically quasinonexpansive mapping of $C$ into itself such that $\bigcap_{i=1}^{\infty} F\left(T_{i}\right) \neq \emptyset$ with $k_{i, n} \geq 1$ and $c_{i, n} \geq 0$ for all $i, n \in \mathbb{N}$ such that $\lim _{n \rightarrow \infty} k_{i, n}=1, \lim _{n \rightarrow \infty} c_{i, n}=0$ and let $W_{n}$ be the $W$-mapping defined by (1.6). Then the followings hold:

(i) There exist $\left\{\gamma_{1, n}\right\} \subset[1, \infty)$ and $\left\{\varepsilon_{1, n}\right\} \subset[0, \infty)$ such that $\left\|W_{n} x-p\right\| \leq$ $\gamma_{1, n}\|x-p\|+\varepsilon_{1, n}$ for all $x \in C, p \in \bigcap_{i=1}^{\infty} F\left(T_{i}\right)$ and all $n \in \mathbb{N}$.

(ii) There exists a constant $L^{*}>0$ such that

$$
\left\|W_{n} x-W_{n} y\right\| \leq L^{*}\|x-y\|
$$

for all $x, y \in C$ and $n \in \mathbb{N}$.

(iii) If $0<\ell \leq \alpha_{i, n} \leq k<1$ for all $n \in \mathbb{N}$ and $i=1,2, \ldots, n$ for some $\ell, k$ with $0<\ell<k<1$ and $\lim _{n \rightarrow \infty} \gamma_{1, n}=1, \lim _{n \rightarrow \infty} \varepsilon_{1, n}=0$, then for each bounded sequence $\left\{x_{n}\right\}$ in $C$, we have

$$
\begin{aligned}
& \lim _{n \rightarrow \infty}\left\|x_{n+1}-x_{n}\right\| \\
= & \lim _{n \rightarrow \infty}\left\|W_{n} x_{n}-x_{n}\right\|=0 \Longrightarrow \lim _{n \rightarrow \infty}\left\|T_{i} x_{n}-x_{n}\right\|=0 \quad \forall i=1,2, \ldots, n .
\end{aligned}
$$

(iv) If $0<\ell \leq \alpha_{i, n} \leq k<1$ for all $n \in \mathbb{N}$ and $i=1,2, \ldots, n$ for some $\ell, k$ with $0<\ell<k<1$ and $\lim _{n \rightarrow \infty} \gamma_{1, n}=1, \lim _{n \rightarrow \infty} \varepsilon_{1, n}=0$, then $\bigcap_{i=1}^{\infty} F\left(T_{i}\right)=$ $\bigcap_{n=1}^{\infty} F\left(W_{n}\right)$. 
Proof. (i) Let $n \in \mathbb{N}, x \in C$ and $p \in \bigcap_{i=1}^{\infty} F\left(T_{i}\right)$. We have for each $i=$ $1,2, \ldots, n$ that

$$
\begin{aligned}
& \left\|W_{n} x-p\right\|=\left\|U_{1, n} x-p\right\| \\
& =\left\|\alpha_{1, n} T_{1}^{n} U_{2, n} x+\left(1-\alpha_{1, n}\right) x-p\right\| \\
& \leq \alpha_{1, n}\left\|T_{1}^{n} U_{2, n} x-p\right\|+\left(1-\alpha_{1, n}\right)\|x-p\| \\
& \leq \alpha_{1, n} k_{1, n}\left\|U_{2, n} x-p\right\|+\alpha_{1, n} c_{1, n}+\left(1-\alpha_{1, n}\right)\|x-p\| \\
& \leq \alpha_{1, n} k_{1, n}\left\|\alpha_{2, n} T_{2}^{n} U_{3, n} x+\left(1-\alpha_{2, n}\right) x-p\right\|+\alpha_{1, n} c_{1, n} \\
& +\left(1-\alpha_{1, n}\right)\|x-p\| \\
& \leq \alpha_{1, n} k_{1, n} \alpha_{2, n}\left\|T_{2}^{n} U_{3, n} x-p\right\|+\alpha_{1, n} k_{1, n}\left(1-\alpha_{2, n}\right)\|x-p\| \\
& +\alpha_{1, n} c_{1, n}+\left(1-\alpha_{1, n}\right)\|x-p\| \\
& \leq \alpha_{1, n} k_{1, n} \alpha_{2, n} k_{2, n}\left\|U_{3, n} x-p\right\| \\
& +\left(\alpha_{1, n} k_{1, n}\left(1-\alpha_{2, n}\right)+\left(1-\alpha_{1, n}\right)\right)\|x-p\| \\
& +\left(\alpha_{1, n} k_{1, n} \alpha_{2, n} c_{2, n}+\alpha_{1, n} c_{1, n}\right) \\
& \vdots \\
& \leq \alpha_{1, n} k_{1, n} \alpha_{2, n} k_{2, n} \cdots \alpha_{n-1, n} k_{n-1, n}\left\|U_{n, n} x-p\right\| \\
& +\left(\alpha_{1, n} k_{1, n} \alpha_{2, n} k_{2, n} \cdots \alpha_{n-2, n} k_{n-2, n}\left(1-\alpha_{n-1, n}\right)\right. \\
& +\alpha_{1, n} k_{1, n} \alpha_{2, n} k_{2, n} \cdots \alpha_{n-3, n} k_{n-3, n}\left(1-\alpha_{n-2, n}\right) \\
& \left.+\cdots+\alpha_{1, n} k_{1, n}\left(1-\alpha_{2, n}\right)+\left(1-\alpha_{1, n}\right)\right)\|x-p\| \\
& +\left(\alpha_{1, n} k_{1, n} \alpha_{2, n} k_{2, n} \cdots \alpha_{n-1, n} c_{n-1, n}\right. \\
& +\alpha_{1, n} k_{1, n} \alpha_{2, n} k_{2, n} \cdots \alpha_{n-2, n} c_{n-2, n} \\
& \left.+\cdots+\alpha_{1, n} k_{1, n} \alpha_{2, n} c_{2, n}+\alpha_{1, n} c_{1, n}\right) \\
& =\alpha_{1, n} k_{1, n} \alpha_{2, n} k_{2, n} \cdots \alpha_{n-1, n} k_{n-1, n}\left\|\alpha_{n, n} T_{n}^{n} x+\left(1-\alpha_{n, n}\right) x-p\right\| \\
& +\left(\alpha_{1, n} k_{1, n} \alpha_{2, n} k_{2, n} \cdots \alpha_{n-2, n} k_{n-2, n}\left(1-\alpha_{n-1, n}\right)\right. \\
& +\alpha_{1, n} k_{1, n} \alpha_{2, n} k_{2, n} \cdots \alpha_{n-3, n} k_{n-3, n}\left(1-\alpha_{n-2, n}\right) \\
& \left.+\cdots+\alpha_{1, n} k_{1, n}\left(1-\alpha_{2, n}\right)+\left(1-\alpha_{1, n}\right)\right)\|x-p\| \\
& +\left(\alpha_{1, n} k_{1, n} \alpha_{2, n} k_{2, n} \cdots \alpha_{n-1, n} c_{n-1, n}\right. \\
& +\alpha_{1, n} k_{1, n} \alpha_{2, n} k_{2, n} \cdots \alpha_{n-2, n} c_{n-2, n} \\
& \left.+\cdots+\alpha_{1, n} k_{1, n} \alpha_{2, n} c_{2, n}+\alpha_{1, n} c_{1, n}\right) \\
& \leq \alpha_{1, n} k_{1, n} \alpha_{2, n} k_{2, n} \cdots \alpha_{n-1, n} k_{n-1, n} \alpha_{n, n}\left\|T_{n}^{n} x-p\right\| \\
& +\left(\alpha_{1, n} k_{1, n} \alpha_{2, n} k_{2, n} \cdots \alpha_{n-1, n} k_{n-1, n}\left(1-\alpha_{n, n}\right)\right. \\
& +\alpha_{1, n} k_{1, n} \alpha_{2, n} k_{2, n} \cdots \alpha_{n-2, n} k_{n-2, n}\left(1-\alpha_{n-1, n}\right) \\
& \left.+\cdots+\alpha_{1, n} k_{1, n}\left(1-\alpha_{2, n}\right)+\left(1-\alpha_{1, n}\right)\right)\|x-p\| \\
& +\left(\alpha_{1, n} k_{1, n} \alpha_{2, n} k_{2, n} \cdots \alpha_{n-1, n} c_{n-1, n}\right. \\
& +\alpha_{1, n} k_{1, n} \alpha_{2, n} k_{2, n} \cdots \alpha_{n-2, n} c_{n-2, n} \\
& \left.+\cdots+\alpha_{1, n} k_{1, n} \alpha_{2, n} c_{2, n}+\alpha_{1, n} c_{1, n}\right)
\end{aligned}
$$




$$
\begin{aligned}
\leq & \left(\alpha_{1, n} k_{1, n} \alpha_{2, n} k_{2, n} \cdots \alpha_{n, n} k_{n, n}\right. \\
& +\alpha_{1, n} k_{1, n} \alpha_{2, n} k_{2, n} \cdots \alpha_{n-1, n} k_{n-1, n}\left(1-\alpha_{n, n}\right) \\
& +\alpha_{1, n} k_{1, n} \alpha_{2, n} k_{2, n} \cdots \alpha_{n-2, n} k_{n-2, n}\left(1-\alpha_{n-1, n}\right) \\
& \left.+\cdots+\alpha_{1, n} k_{1, n}\left(1-\alpha_{2, n}\right)+\left(1-\alpha_{1, n}\right)\right)\|x-p\| \\
& +\left(\alpha_{1, n} k_{1, n} \alpha_{2, n} k_{2, n} \cdots \alpha_{n, n} c_{n, n}+\alpha_{1, n} k_{1, n} \alpha_{2, n} k_{2, n} \cdots \alpha_{n-1, n} c_{n-1, n}\right. \\
& \left.+\cdots+\alpha_{1, n} k_{1, n} \alpha_{2, n} c_{2, n}+\alpha_{1, n} c_{1, n}\right) \\
= & \left(\alpha_{1, n} k_{1, n} \alpha_{2, n} k_{2, n} \cdots \alpha_{n, n}\left(k_{n, n}-1\right)\right. \\
& +\alpha_{1, n} k_{1, n} \alpha_{2, n} k_{2, n} \cdots \alpha_{n-1, n}\left(k_{n-1, n}-1\right) \\
& \left.+\cdots+\alpha_{1, n}\left(k_{1, n}-1\right)+1\right)\|x-p\| \\
& +\left(\alpha_{1, n} k_{1, n} \alpha_{2, n} k_{2, n} \cdots \alpha_{n, n} c_{n, n}+\alpha_{1, n} k_{1, n} \alpha_{2, n} k_{2, n} \cdots \alpha_{n-1, n} c_{n-1, n}\right. \\
& \left.+\cdots+\alpha_{1, n} k_{1, n} \alpha_{2, n} c_{2, n}+\alpha_{1, n} c_{1, n}\right) .
\end{aligned}
$$

Put

$$
\begin{aligned}
\gamma_{1, n}= & \alpha_{1, n} k_{1, n} \alpha_{2, n} k_{2, n} \cdots \alpha_{n, n}\left(k_{n, n}-1\right) \\
& +\alpha_{1, n} k_{1, n} \alpha_{2, n} k_{2, n} \cdots \alpha_{n-1, n}\left(k_{n-1, n}-1\right) \\
& +\cdots+\alpha_{1, n}\left(k_{1, n}-1\right)+1
\end{aligned}
$$

and

$$
\begin{aligned}
\varepsilon_{1, n}= & \alpha_{1, n} k_{1, n} \alpha_{2, n} k_{2, n} \cdots \alpha_{n, n} c_{n, n} \\
& +\alpha_{1, n} k_{1, n} \alpha_{2, n} k_{2, n} \cdots \alpha_{n-1, n} c_{n-1, n} \\
& +\cdots+\alpha_{1, n} k_{1, n} \alpha_{2, n} c_{2, n}+\alpha_{1, n} c_{1, n} .
\end{aligned}
$$

Then $\gamma_{1, n} \geq 1, \varepsilon_{1, n} \geq 0$ and $\left\|W_{n} x-p\right\| \leq \gamma_{1, n}\|x-p\|+\varepsilon_{1, n}$.

(ii) Let $n \in \mathbb{N}$ and $x, y \in C$, we have

$$
\begin{aligned}
\left\|W_{n} x-W_{n} y\right\| \leq & \alpha_{1, n}\left\|T_{1}^{n} U_{2, n} x-T_{1}^{n} U_{2, n} y\right\|+\left(1-\alpha_{1, n}\right)\|x-y\| \\
\leq & \alpha_{1, n} L_{1}\left\|U_{2, n} x-U_{2, n} y\right\|+\left(1-\alpha_{1, n}\right)\|x-y\| \\
\leq & \alpha_{1, n} L_{1} \alpha_{2, n}\left\|T_{2}^{n} U_{3, n} x-T_{2}^{n} U_{3, n} y\right\| \\
& +\left(\alpha_{1, n} L_{1}\left(1-\alpha_{2, n}\right)+\left(1-\alpha_{1, n}\right)\right)\|x-y\| \\
\leq & \alpha_{1, n} L_{1} \alpha_{2, n} L_{2}\left\|U_{3, n} x-U_{3, n} y\right\| \\
& \quad+\left(\alpha_{1, n} L_{1}\left(1-\alpha_{2, n}\right)+\left(1-\alpha_{1, n}\right)\right)\|x-y\| \\
& \vdots \\
\leq & \alpha_{1, n} L_{1} \alpha_{2, n} L_{2} \cdots \alpha_{n-1, n} L_{n-1}\left\|U_{n, n} x-U_{n, n} y\right\| \\
& +\left(\alpha_{1, n} L_{1} \alpha_{2, n} L_{2} \cdots \alpha_{n-2, n} L_{n-2}\left(1-\alpha_{n-1, n}\right)\right. \\
& +\alpha_{1, n} L_{1} \alpha_{2, n} L_{2} \cdots \alpha_{n-3, n} L_{n-3}\left(1-\alpha_{n-2, n}\right) \\
& \left.+\cdots+\alpha_{1, n} L_{1}\left(1-\alpha_{2, n}\right)+\left(1-\alpha_{1, n}\right)\right)\|x-y\|
\end{aligned}
$$




$$
\begin{aligned}
\leq & \alpha_{1, n} L_{1} \cdots \alpha_{n-1, n} L_{n-1}\left(\alpha_{n, n}\left\|T_{n}^{n} x-T_{n}^{n} y\right\|+\left(1-\alpha_{n, n}\right)\|x-y\|\right) \\
& +\left(\alpha_{1, n} L_{1} \alpha_{2, n} L_{2} \cdots \alpha_{n-2, n} L_{n-2}\left(1-\alpha_{n-1, n}\right)\right. \\
& +\alpha_{1, n} L_{1} \alpha_{2, n} L_{2} \cdots \alpha_{n-3, n} L_{n-3}\left(1-\alpha_{n-2, n}\right) \\
& \left.+\cdots+\alpha_{1, n} L_{1}\left(1-\alpha_{2, n}\right)+\left(1-\alpha_{1, n}\right)\right)\|x-y\| \\
\leq & \left(\alpha_{1, n} L_{1} \alpha_{2, n} L_{2} \cdots \alpha_{n, n} L_{n}\right. \\
& +\alpha_{1, n} L_{1} \alpha_{2, n} L_{2} \cdots \alpha_{n-1, n} L_{n-1}\left(1-\alpha_{n, n}\right) \\
& +\alpha_{1, n} L_{1} \alpha_{2, n} L_{2} \cdots \alpha_{n-2, n} L_{n-2}\left(1-\alpha_{n-1, n}\right) \\
& +\alpha_{1, n} L_{1} \alpha_{2, n} L_{2} \cdots \alpha_{n-3, n} L_{n-3}\left(1-\alpha_{n-2, n}\right) \\
& \left.+\cdots+\alpha_{1, n} L_{1}\left(1-\alpha_{2, n}\right)+\left(1-\alpha_{1, n}\right)\right)\|x-y\| . \\
\leq & \left(L_{1} L_{2} \cdots L_{n}+L_{1} L_{2} \cdots L_{n-1}\right. \\
& \left.+L_{1} L_{2} \cdots L_{n-2}+L_{1} L_{2} \cdots L_{n-3}+\cdots+L_{1}+1\right)\|x-y\| .
\end{aligned}
$$

Put

$$
\begin{aligned}
L^{*}= & L_{1} L_{2} \cdots L_{n}+L_{1} L_{2} \cdots L_{n-1}+L_{1} L_{2} \cdots L_{n-2}+L_{1} L_{2} \cdots L_{n-3} \\
& +\cdots+L_{1}+1 .
\end{aligned}
$$

It is easy to see that $L^{*}>0$. Hence (ii) is proved.

(iii) Let $\left\{x_{n}\right\} \subset C$ be a bounded sequence and $\lim _{n \rightarrow \infty}\left\|W_{n} x_{n}-x_{n}\right\|=0$. First, as $n \rightarrow \infty$, we observe

$$
\left\|x_{n}-T_{1}^{n} U_{2, n} x_{n}\right\|=\frac{1}{\alpha_{1, n}}\left\|W_{n} x_{n}-x_{n}\right\| \leq \frac{1}{\ell}\left\|W_{n} x_{n}-x_{n}\right\| \rightarrow 0 .
$$

Let $p \in \bigcap_{i=1}^{\infty} F\left(T_{i}\right)$. Put $M=\sup _{n \in N}\left\{\left\|x_{n}-T_{1}^{n} U_{2, n} x_{n}\right\|+2\left\|T_{1}^{n} U_{2, n} x_{n}-p\right\|\right\}$ and $r=\sup _{n \in \mathbb{N}}\left\{\left\|T_{2}^{n} U_{3, n} x_{n}-p\right\|\right\}+\sup _{n \in \mathbb{N}}\left\{\left\|x_{n}-p\right\|\right\}$. By Lemma 2.7, there is a continuous strictly increasing convex function $g:[0, \infty) \rightarrow[0, \infty)$ with $g(0)=0$ such that

$$
\begin{aligned}
\left\|x_{n}-p\right\|^{2} \leq & \left(\left\|x_{n}-T_{1}^{n} U_{2, n} x_{n}\right\|+\left\|T_{1}^{n} U_{2, n} x_{n}-p\right\|\right)^{2} \\
= & \left\|x_{n}-T_{1}^{n} U_{2, n} x_{n}\right\|\left(\left\|x_{n}-T_{1}^{n} U_{2, n} x_{n}\right\|+2\left\|T_{1}^{n} U_{2, n} x_{n}-p\right\|\right) \\
& +\left\|T_{1}^{n} U_{2, n} x_{n}-p\right\|^{2} \\
\leq & M\left\|x_{n}-T_{1}^{n} U_{2, n} x_{n}\right\|+\left\|T_{1}^{n} U_{2, n} x_{n}-p\right\|^{2} \\
\leq & M\left\|x_{n}-T_{1}^{n} U_{2, n} x_{n}\right\|+k_{1, n}^{2}\left\|U_{2, n} x_{n}-p\right\|^{2} \\
& +2 k_{1, n} c_{1, n}\left\|U_{2, n} x_{n}-p\right\|+c_{1, n}^{2} \\
\leq & M\left\|x_{n}-T_{1}^{n} U_{2, n} x_{n}\right\|+k_{1, n}^{2}\left\|\alpha_{2, n} T_{2}^{n} U_{3, n} x_{n}+\left(1-\alpha_{2, n}\right) x_{n}-p\right\|^{2} \\
& +2 k_{1, n} c_{1, n}\left\|U_{2, n} x_{n}-p\right\|+c_{1, n}^{2}
\end{aligned}
$$




$$
\begin{aligned}
\leq & M\left\|x_{n}-T_{1}^{n} U_{2, n} x_{n}\right\|+k_{1, n}^{2} \alpha_{2, n}\left\|T_{2}^{n} U_{3, n} x_{n}-p\right\|^{2} \\
& +k_{1, n}^{2}\left(1-\alpha_{2, n}\right)\left\|x_{n}-p\right\|^{2}+2 k_{1, n} c_{1, n}\left\|U_{2, n} x_{n}-p\right\|+c_{1, n}^{2} \\
& -k_{1, n}^{2} \alpha_{2, n}\left(1-\alpha_{2, n}\right) g\left(\left\|T_{2}^{n} U_{3, n} x_{n}-x_{n}\right\|\right) \\
\leq & M\left\|x_{n}-T_{1}^{n} U_{2, n} x_{n}\right\|+k_{1, n}^{2} \alpha_{2, n} k_{2, n}^{2}\left\|U_{3, n} x_{n}-p\right\|^{2} \\
& +2 k_{1, n}^{2} \alpha_{2, n} k_{2, n} c_{2, n}\left\|U_{3, n} x_{n}-p\right\|+k_{1, n}^{2} \alpha_{2, n} c_{2, n}^{2} \\
& +k_{1, n}^{2}\left(1-\alpha_{2, n}\right)\left\|x_{n}-p\right\|^{2}+2 k_{1, n} c_{1, n}\left\|U_{2, n} x_{n}-p\right\|+c_{1, n}^{2} \\
& -k_{1, n}^{2} \alpha_{2, n}\left(1-\alpha_{2, n}\right) g\left(\left\|T_{2}^{n} U_{3, n} x_{n}-x_{n}\right\|\right) \\
\leq & M\left\|x_{n}-T_{1}^{n} U_{2, n} x_{n}\right\|+k_{1, n}^{2} \alpha_{2, n} k_{2, n}^{2} \gamma_{3, n}^{2}\left\|x_{n}-p\right\|^{2} \\
& +2 k_{1, n}^{2} \alpha_{2, n} k_{2, n}^{2} \gamma_{3, n} \varepsilon_{3, n}\left\|x_{n}-p\right\|+k_{1, n}^{2} \alpha_{2, n} k_{2, n}^{2} \varepsilon_{3, n}^{2} \\
& +2 k_{1, n}^{2} \alpha_{2, n} k_{2, n} c_{2, n}\left\|U_{3, n} x_{n}-p\right\|+k_{1, n}^{2} \alpha_{2, n} c_{2, n}^{2} \\
& +k_{1, n}^{2}\left(1-\alpha_{2, n}\right)\left\|x_{n}-p\right\|^{2}+2 k_{1, n} c_{1, n}\left\|U_{2, n} x_{n}-p\right\|+c_{1, n}^{2} \\
& -k_{1, n}^{2} \alpha_{2, n}\left(1-\alpha_{2, n}\right) g\left(\left\|T_{2}^{n} U_{3, n} x_{n}-x_{n}\right\|\right) .
\end{aligned}
$$

It follows that

$$
\begin{aligned}
& k_{1, n}^{2} \alpha_{2, n}\left(1-\alpha_{2, n}\right) g\left(\left\|T_{2}^{n} U_{3, n} x_{n}-x_{n}\right\|\right) \\
\leq & M\left\|x_{n}-T_{1}^{n} U_{2, n} x_{n}\right\| \\
& +2 k_{1, n}^{2} \alpha_{2, n} k_{2, n}^{2} \gamma_{3, n} \varepsilon_{3, n}\left\|x_{n}-p\right\|+k_{1, n}^{2} \alpha_{2, n} k_{2, n}^{2} \varepsilon_{3, n}^{2} \\
& +2 k_{1, n}^{2} \alpha_{2, n} k_{2, n} c_{2, n}\left\|U_{3, n} x_{n}-p\right\|+k_{1, n}^{2} \alpha_{2, n} c_{2, n}^{2} \\
& +2 k_{1, n} c_{1, n}\left\|U_{2, n} x_{n}-p\right\|+c_{1, n}^{2} \\
& +\left(k_{1, n}^{2} \alpha_{2, n} k_{2, n}^{2} \gamma_{3, n}^{2}+k_{1, n}^{2}\left(1-\alpha_{2, n}\right)-1\right)\left\|x_{n}-p\right\|^{2} .
\end{aligned}
$$

Since $\lim _{n \rightarrow \infty}\left\|x_{n}-T_{1}^{n} U_{2, n} x_{n}\right\|=0, \lim _{n \rightarrow \infty} \gamma_{3, n}=1, \lim _{n \rightarrow \infty} \varepsilon_{3, n}=0$ and $0<\ell \leq \alpha_{2, n} \leq k<1$, we obtain that $\lim _{n \rightarrow \infty} g\left(\left\|T_{2}^{n} U_{3, n} x_{n}-x_{n}\right\|\right)=0$. By the property of $g$, it follows from (3.3) that $\lim _{n \rightarrow \infty}\left\|T_{2}^{n} U_{3, n} x_{n}-x_{n}\right\|=0$. Similarly, we can show that

$$
\lim _{n \rightarrow \infty}\left\|T_{i}^{n} U_{i+1, n} x_{n}-x_{n}\right\|=0
$$

for all $i \in \mathbb{N}$. This implies for each $i=1,2, \ldots, n$ that

$$
\begin{aligned}
\left\|U_{i, n} x_{n}-x_{n}\right\| & =\left\|\alpha_{i, n} T_{i}^{n} U_{i+1, n} x_{n}+\left(1-\alpha_{i, n}\right) x_{n}-x_{n}\right\| \\
& =\alpha_{i, n}\left\|T_{i}^{n} U_{i+1, n} x_{n}-x_{n}\right\| \rightarrow 0
\end{aligned}
$$

as $n \rightarrow \infty$. We observe that

$$
\text { (3.6) } \begin{aligned}
\left\|x_{n}-T_{i}^{n} x_{n}\right\| & \leq\left\|x_{n}-T_{i}^{n} U_{i+1, n} x_{n}\right\|+\left\|T_{i}^{n} U_{i+1, n} x_{n}-T_{i}^{n} x_{n}\right\| \\
& \leq\left\|x_{n}-T_{i}^{n} U_{i+1, n} x_{n}\right\|+L_{i}\left\|U_{i+1, n} x_{n}-x_{n}\right\|
\end{aligned}
$$

for all $i=1,2, \ldots, n$. From (3.4), (3.5) and (3.6), we have

$$
\lim _{n \rightarrow \infty}\left\|x_{n}-T_{i}^{n} x_{n}\right\|=0 .
$$

Lemma 2.8 yields that $\lim _{n \rightarrow \infty}\left\|x_{n}-T_{i} x_{n}\right\|=0$. 
(iv) It is easy to see that $\bigcap_{i=1}^{\infty} F\left(T_{i}\right) \subset \bigcap_{n=1}^{\infty} F\left(W_{n}\right)$. Let $z \in \bigcap_{n=1}^{\infty} F\left(W_{n}\right)$. Then $\left\|W_{n} z-z\right\|=0$ for all $n \in \mathbb{N}$. By (iii), we have $z=T_{i} z$ for all $i=$ $1,2, \ldots, n$. So we have $\bigcap_{i=1}^{\infty} F\left(T_{i}\right)=\bigcap_{n=1}^{\infty} F\left(W_{n}\right)$.

\section{Strong convergence theorems}

In this section, we prove strong convergence theorems for finding a common element of the solutions set of an equilibrium problem and the fixed points set of a countable family of generalized asymptotically quasi-nonexpansive and uniformly Lipschitzian mappings.

Theorem 4.1. Let $C$ be a closed and convex subset of a real Hilbert space $H$, let $F: C \times C \rightarrow R$ be a bifunction satisfying (A1) - (A4) and let $\left\{T_{i}\right\}_{i=1}^{\infty}$ be a family of uniformly $L_{i}$-Lipschitzian and generalized quasi-nonexpansive mappings of $C$ into itself. Assume that $\Omega:=\bigcap_{i=1}^{\infty} F\left(T_{i}\right) \cap E P(F) \neq \emptyset$ and $\left\{\beta_{n}\right\} \subset[0,1)$ such that $\lim _{\sup _{n \rightarrow \infty}} \beta_{n}<1$. Let $W_{n}$ be the $W$-mapping generated by $T_{n}, T_{n-1}, \ldots, T_{1}$ and $\alpha_{n, n}, \alpha_{n-1, n}, \ldots, \alpha_{1, n}$ with $0<\ell \leq \alpha_{i, n} \leq k<1$ for all $n \in \mathbb{N}$ and $i=1,2, \ldots, n$. Let $\left\{\gamma_{1, n}\right\} \subset[1, \infty),\left\{\varepsilon_{1, n}\right\} \subset[0, \infty)$ be as in Lemma 3.1(i) with $\lim _{n \rightarrow \infty} \gamma_{1, n}=1$ and $\lim _{n \rightarrow \infty} \varepsilon_{1, n}=0$. For an initial point $x_{0} \in H$ with $C_{1}=C$ and $x_{1}=P_{C_{1}} x_{0}$, let $\left\{x_{n}\right\},\left\{y_{n}\right\}$ and $\left\{u_{n}\right\}$ be sequences generated by

$$
\left\{\begin{array}{l}
F\left(u_{n}, y\right)+\frac{1}{r_{n}}\left\langle y-u_{n}, u_{n}-x_{n}\right\rangle \geq 0, \quad \forall y \in C \\
y_{n}=\beta_{n} u_{n}+\left(1-\beta_{n}\right) W_{n} u_{n} \\
C_{n+1}=\left\{z \in C_{n}:\left\|y_{n}-z\right\|^{2}\right. \\
\left.\quad \leq\left\|x_{n}-z\right\|^{2}-\beta_{n}\left(1-\beta_{n}\right)\left\|W_{n} x_{n}-x_{n}\right\|^{2}+\left(1-\beta_{n}\right) \theta_{n}\right\} \\
x_{n+1}=P_{C_{n+1}} x_{0}, \quad n \in \mathbb{N},
\end{array}\right.
$$

where $\theta_{n}=\left(\gamma_{1, n}^{2}-1\right) \nabla_{n}^{2}+2 \gamma_{1, n} \varepsilon_{1, n} \nabla_{n}+\varepsilon_{1, n}^{2}, \nabla_{n}=\sup _{n \in \mathbb{N}}\left\{\left\|x_{n}-z\right\|: z \in\right.$ $\Omega\}<\infty$. Then the sequence $\left\{x_{n}\right\}$ converges strongly to $z_{0}=P_{\Omega} x_{0}$.

Proof. We split the proof into six steps.

Step 1. Show that $\Omega \subset C_{n}$ for all $n \geq 1$.

By Lemma 2.9, we know that $\bigcap_{i=1}^{\infty} F\left(T_{i}\right)$ is closed and convex. Moreover, by Lemma 2.4, $C_{n}$ is also closed and convex for each $n \in \mathbb{N}$. $\Omega \subset C_{1}=C$ is obviuos. Suppose that $\Omega \subset C_{k}$ for $k \geq 1$. For each $p \in \Omega$, by Lemma 3.1(i), we see that

$$
\begin{aligned}
\left\|y_{k}-p\right\|^{2}= & \left\|\beta_{k} x_{k}+\left(1-\beta_{k}\right) W_{k} x_{k}-p\right\|^{2} \\
= & \beta_{k}\left\|x_{k}-p\right\|^{2}+\left(1-\beta_{k}\right)\left\|W_{k} x_{k}-p\right\|^{2}-\beta_{k}\left(1-\beta_{k}\right)\left\|W_{k} x_{k}-x_{k}\right\|^{2} \\
\leq & \beta_{k}\left\|x_{k}-p\right\|^{2}+\left(1-\beta_{k}\right)\left(\gamma_{1, k}^{2}\left\|x_{k}-p\right\|^{2}+2 \gamma_{1, k} \varepsilon_{1, k}\left\|x_{k}-p\right\|+\varepsilon_{1, k}^{2}\right) \\
& \quad-\beta_{k}\left(1-\beta_{k}\right)\left\|W_{k} x_{k}-x_{k}\right\|^{2} \\
= & \left\|x_{k}-p\right\|^{2}-\beta_{k}\left(1-\beta_{k}\right)\left\|W_{k} x_{k}-x_{k}\right\|^{2} \\
& \quad+\left(1-\beta_{k}\right)\left(\left(\gamma_{1, k}^{2}-1\right)\left\|x_{k}-p\right\|^{2}+2 \gamma_{1, k} \varepsilon_{1, k}\left\|x_{k}-p\right\|+\varepsilon_{1, k}^{2}\right) \\
\leq & \left\|x_{k}-p\right\|^{2}-\beta_{k}\left(1-\beta_{k}\right)\left\|W_{k} x_{k}-x_{k}\right\|^{2}+\left(1-\beta_{k}\right) \theta_{k} .
\end{aligned}
$$


So we have $p \in C_{k+1}$. By induction, we have $\Omega \subset C_{n}$ for all $n \in \mathbb{N}$.

Step 2. Show that $\lim _{n \rightarrow \infty}\left\|x_{n}-x_{0}\right\|$ exists.

Since $\Omega$ is a nonempty, closed and convex subset of $C$, there exists a unique element $z_{0}=P_{\Omega} x_{0} \in \Omega \subset C_{n}$. Since $x_{n}=P_{C_{n}} x_{0}$,

$$
\left\|x_{n}-x_{0}\right\| \leq\left\|z_{0}-x_{0}\right\| \text {. }
$$

Hence $\left\{x_{n}\right\}$ is bounded. So are $\left\{y_{n}\right\}$ and $\left\{u_{n}\right\}$. Since $x_{n+1}=P_{C_{n+1}} x_{0} \in$ $C_{n+1} \subset C_{n}$,

$$
\left\|x_{n}-x_{0}\right\| \leq\left\|x_{n+1}-x_{0}\right\|
$$

From (4.1) and (4.2), we get that $\lim _{n \rightarrow \infty}\left\|x_{n}-x_{0}\right\|$ exists.

Step 3. Show that $\left\{x_{n}\right\}$ is a Cauchy sequence.

By the construction of the set $C_{n}$, we know that $x_{m}=P_{C_{m}} x_{0} \in C_{m} \subset C_{n}$ for $m>n$. By Lemma 2.2, it follows that

$$
\left\|x_{m}-x_{n}\right\|^{2} \leq\left\|x_{m}-x_{0}\right\|^{2}-\left\|x_{n}-x_{0}\right\|^{2} \rightarrow 0
$$

as $m, n \rightarrow \infty$. Hence $\left\{x_{n}\right\}$ is a Cauchy sequence in $C$. So $x_{n} \rightarrow q \in C$ as $n \rightarrow \infty$.

Step 4. Show that $q \in \bigcap_{i=1}^{\infty} F\left(T_{i}\right)$.

From (4.3), we get

$$
\left\|x_{n+1}-x_{n}\right\| \rightarrow 0
$$

as $n \rightarrow \infty$. Since $x_{n+1} \in C_{n+1} \subset C_{n}$,

$$
\begin{aligned}
\left\|y_{n}-x_{n+1}\right\|^{2} & \leq\left\|x_{n}-x_{n+1}\right\|^{2}-\beta_{n}\left(1-\beta_{n}\right)\left\|W_{n} x_{n}-x_{n}\right\|^{2}+\left(1-\beta_{n}\right) \theta_{n} \\
& \leq\left\|x_{n}-x_{n+1}\right\|^{2}+\left(1-\beta_{n}\right) \theta_{n} \rightarrow 0
\end{aligned}
$$

as $n \rightarrow \infty$, which implies

$$
\left\|y_{n}-x_{n}\right\| \leq\left\|y_{n}-x_{n+1}\right\|+\left\|x_{n+1}-x_{n}\right\| \rightarrow 0
$$

as $n \rightarrow \infty$. Since $T_{r_{n}}$ is firmly nonexpansive, for $p \in \Omega$, we have

$$
\left\|u_{n}-p\right\|=\left\|T_{r_{n}} x_{n}-T_{r_{n}} p\right\| \leq\left\|x_{n}-p\right\|
$$

and

$$
\begin{aligned}
& \left\|u_{n}-p\right\|^{2}=\left\|T_{r_{n}} x_{n}-T_{r_{n}} p\right\|^{2} \\
\leq & \left\langle T_{r_{n}} x_{n}-T_{r_{n}} p, x_{n}-p\right\rangle \\
= & \left\langle u_{n}-p, x_{n}-p\right\rangle \\
= & \frac{1}{2}\left\{\left\|u_{n}-p\right\|^{2}+\left\|x_{n}-p\right\|^{2}-\left\|x_{n}-u_{n}\right\|^{2}\right\},
\end{aligned}
$$

hence

$$
\left\|u_{n}-p\right\|^{2} \leq\left\|x_{n}-p\right\|^{2}-\left\|x_{n}-u_{n}\right\|^{2}
$$


It follows from (4.5) and (4.6) that

$$
\begin{aligned}
& \left\|y_{n}-p\right\|^{2} \\
\leq & \beta_{n}\left\|u_{n}-p\right\|^{2}+\left(1-\beta_{n}\right)\left\|W_{n} u_{n}-p\right\|^{2} \\
\leq & \beta_{n}\left\|x_{n}-p\right\|^{2}+\left(1-\beta_{n}\right) \gamma_{1, n}^{2}\left\|u_{n}-p\right\|^{2}+2 \gamma_{1, n} \varepsilon_{1, n}\left\|x_{n}-p\right\|+\varepsilon_{1, n}^{2} \\
\leq & \beta_{n}\left\|x_{n}-p\right\|^{2}+\left(1-\beta_{n}\right) \gamma_{1, n}^{2}\left(\left\|x_{n}-p\right\|^{2}-\left\|x_{n}-u_{n}\right\|^{2}\right) \\
& +2 \gamma_{1, n} \varepsilon_{1, n}\left\|x_{n}-p\right\|+\varepsilon_{1, n}^{2} \\
\leq & \left(\gamma_{1, n}^{2}-1\right)\left(1-\beta_{n}\right)\left\|x_{n}-p\right\|^{2}+\left\|x_{n}-p\right\|^{2}-\left(1-\beta_{n}\right) \gamma_{1, n}^{2}\left\|x_{n}-u_{n}\right\|^{2} \\
& +2 \gamma_{1, n} \varepsilon_{1, n}\left\|x_{n}-p\right\|+\varepsilon_{1, n}^{2}
\end{aligned}
$$

which yields that

$$
\begin{aligned}
& \left(1-\beta_{n}\right) \gamma_{1, n}^{2}\left\|x_{n}-u_{n}\right\|^{2} \\
\leq & \left(\gamma_{1, n}^{2}-1\right)\left(1-\beta_{n}\right)\left\|x_{n}-p\right\|^{2}+\left\|x_{n}-p\right\|^{2}-\left\|y_{n}-p\right\|^{2} \\
& +2 \gamma_{1, n} \varepsilon_{1, n}\left\|x_{n}-p\right\|+\varepsilon_{1, n}^{2} .
\end{aligned}
$$

Hence, from (4.4), we also have

$$
\lim _{n \rightarrow \infty}\left\|x_{n}-u_{n}\right\|=0
$$

Thus $u_{n} \rightarrow q$ as $n \rightarrow \infty$. From (4.4), (4.7) and $\limsup _{n \rightarrow \infty} \beta_{n}<1$, we have

$$
\left\|W_{n} u_{n}-u_{n}\right\|=\frac{1}{1-\beta_{n}}\left\|y_{n}-u_{n}\right\| \rightarrow 0
$$

as $n \rightarrow \infty$. By Lemma 3.1(iii), we conclude that $q \in \bigcap_{i=1}^{\infty} F\left(T_{i}\right)$.

Step 5. Show that $q \in E P(F)$.

From (4.7) and $\liminf \operatorname{in}_{n \rightarrow \infty} r_{n}>0$, we get

$$
\lim _{n \rightarrow \infty} \frac{1}{r_{n}}\left\|x_{n}-u_{n}\right\|=0 .
$$

From $u_{n}=T_{r_{n}} x_{n}$, we have

$$
F\left(u_{n}, y\right)+\frac{1}{r_{n}}\left\langle y-u_{n}, u_{n}-x_{n}\right\rangle \geq 0, \quad \forall y \in C .
$$

From the monotonicity of $F$, we also have

$$
\frac{1}{r_{n}}\left\langle y-u_{n}, u_{n}-x_{n}\right\rangle \geq F\left(y, u_{n}\right), \quad \forall y \in C,
$$

hence

$$
\left\langle y-u_{n}, \frac{u_{n}-x_{n}}{r_{n}}\right\rangle \geq F\left(y, u_{n}\right), \quad \forall y \in C .
$$

From (4.9) and (A4), we have

$$
0 \geq F(y, q), \quad \forall y \in C .
$$


For $t$ with $0<t \leq 1$ and $y \in C$, let $y_{t}=t y+(1-t) q$. Thus $y_{t} \in C$ and $F\left(y_{t}, q\right) \leq 0$. So we have

$$
0=F\left(y_{t}, y_{t}\right) \leq t F\left(y_{t}, y\right)+(1-t) f\left(y_{t}, q\right) \leq t F\left(y_{t}, y\right)
$$

Dividing by $t$, we obtain

$$
F\left(y_{t}, y\right) \geq 0, \quad \forall y \in C .
$$

Letting $t \downarrow 0$ and from (A3), we get

$$
F(q, y) \geq 0, \quad \forall y \in C .
$$

Therefore $q \in E P(F)$ and hence $q \in \Omega$.

Step 6. Show that $q=P_{\Omega} x_{0}$.

Since $x_{n}=P_{C_{n}} x_{0}$ and $\Omega \subset C_{n}$, we obtain

$$
\left\langle x_{0}-x_{n}, x_{n}-p\right\rangle \geq 0 \quad \forall p \in \Omega .
$$

By taking the limit in (4.10), we obtain

$$
\left\langle x_{0}-q, q-p\right\rangle \geq 0 \quad \forall p \in \Omega .
$$

This shows that $q=P_{\Omega} x_{0}=z_{0}$.

From Step 1 to Step 6, we conclude that $\left\{x_{n}\right\}$ converges strongly to $z_{0}=$ $P_{\Omega} x_{0}$. This completes the proof.

Corollary 4.2. Let $C$ be a closed and convex subset of a real Hilbert space $H$, let $F: C \times C \rightarrow R$ be a bifunction satisfying (A1) - (A4) and let $T$ be a uniformly L-Lipschitzian and generalized quasi-nonexpansive mapping of $C$ into itself with $k_{n} \geq 1$ and $c_{n} \geq 0$ for all $n \in \mathbb{N}$ such that $\lim _{n \rightarrow \infty} k_{n}=1$ and $\lim _{n \rightarrow \infty} c_{n}=0$. Assume that $\Omega:=F(T) \cap E P(F) \neq \emptyset$ and $\left\{\alpha_{n}\right\} \subset[0,1)$ such that $\limsup _{n \rightarrow \infty} \alpha_{n}<1$. For an initial point $x_{0} \in H$ with $C_{1}=C$ and $x_{1}=P_{C_{1}} x_{0}$, let $\left\{x_{n}\right\},\left\{y_{n}\right\}$ and $\left\{u_{n}\right\}$ be sequences generated by

$$
\left\{\begin{array}{l}
F\left(u_{n}, y\right)+\frac{1}{r_{n}}\left\langle y-u_{n}, u_{n}-x_{n}\right\rangle \geq 0, \quad \forall y \in C, \\
y_{n}=\alpha_{n} u_{n}+\left(1-\alpha_{n}\right) T^{n} u_{n}, \\
C_{n+1}=\left\{z \in C_{n}:\left\|y_{n}-z\right\|^{2}\right. \\
\left.\quad \leq\left\|x_{n}-z\right\|^{2}-\alpha_{n}\left(1-\alpha_{n}\right)\left\|T^{n} x_{n}-x_{n}\right\|^{2}+\left(1-\alpha_{n}\right) \theta_{n}\right\}, \\
x_{n+1}=P_{C_{n+1}} x_{0}, \quad n \in \mathbb{N},
\end{array}\right.
$$

where $\theta_{n}=\left(k_{n}^{2}-1\right) \nabla_{n}^{2}+2 k_{n} c_{n} \nabla_{n}+c_{n}^{2}, \nabla_{n}=\sup _{n \in \mathbb{N}}\left\{\left\|x_{n}-z\right\|: z \in \Omega\right\}<\infty$. Then the sequence $\left\{x_{n}\right\}$ converges strongly to $z_{0}=P_{\Omega} x_{0}$.

Proof. Putting, in Theorem 4.1, $\beta_{n}=0, \alpha_{i, n}=\alpha_{n}$ for all $n \in \mathbb{N}, T_{1}=T$ and $T_{i}=I$ for $i=2,3, \ldots, n$, where $I$ is the identity mapping, we obtain the desired result.

Corollary 4.3. Let $C$ be a closed and convex subset of a real Hilbert space $H$ and let $T$ be a uniformly L-Lipschitzian and generalized quasi-nonexpansive mapping of $C$ into itself with $k_{n} \geq 1$ and $c_{n} \geq 0$ such that $\lim _{n \rightarrow \infty} k_{n}=1$ and $\lim _{n \rightarrow \infty} c_{n}=0$. Assume that $F(T) \neq \emptyset$ and $\left\{\alpha_{n}\right\} \subset[0,1)$ with $\limsup _{n \rightarrow \infty} \alpha_{n}$ 
$<1$. For an initial point $x_{0} \in H$ with $C_{1}=C$ and $x_{1}=P_{C_{1}} x_{0}$, let $\left\{x_{n}\right\}$ and $\left\{y_{n}\right\}$ be sequences generated by

$$
\left\{\begin{array}{l}
y_{n}=\alpha_{n} x_{n}+\left(1-\alpha_{n}\right) T^{n} x_{n} \\
C_{n+1}=\left\{z \in C_{n}:\left\|y_{n}-z\right\|^{2}\right. \\
\left.\quad \leq\left\|x_{n}-z\right\|^{2}-\alpha_{n}\left(1-\alpha_{n}\right)\left\|T^{n} x_{n}-x_{n}\right\|^{2}+\left(1-\alpha_{n}\right) \theta_{n}\right\} \\
x_{n+1}=P_{C_{n+1}} x_{0}, \quad n \in \mathbb{N},
\end{array}\right.
$$

where $\theta_{n}=\left(k_{n}^{2}-1\right) \nabla_{n}^{2}+2 k_{n} c_{n} \nabla_{n}+c_{n}^{2}, \nabla_{n}=\sup _{n \in \mathbb{N}}\left\{\left\|x_{n}-z\right\|: z \in F(T)\right\}<$ $\infty$. Then the sequence $\left\{x_{n}\right\}$ converges strongly to $z_{0}=P_{F(T)} x_{0}$.

Proof. Put $F(x, y)=0$ for all $x, y \in C$ in Corollary 4.2 .

Acknowledgement. The authors wish to thank the Thailand Research Fund, Thailand. P. Cholamjiak is supported by the Thailand Research Fund, the Commission on Higher Education, and University of Phayao under Grant MRG5580016.

\section{References}

[1] H. H. Bauschke, E. Matoušková, and S. Reich, Projection and proximal point methods: convergence results and counterexamples, Nonlinear Anal. 56 (2004), no. 5, 715-738.

[2] E. Blum and W. Oettli, From optimization and variational inequalities to equilibrium problems, Math. Student 63 (1994), no. 1-4, 123-145.

[3] L. C. Ceng and J. C. Yao, A hybrid iterative scheme for mixed equilibrium problems and fixed point problems, J. Comput. Appl. Math. 214 (2008), no. 1, 186-201.

[4] J. W. Chen, Y. J. Cho, and Z. Wan, Shrinking projection algorithms for equilibrium problems with a bifunction defined on the dual space of a Banach space, Fixed Point Theory Appl. 2011 (2011), 11 pages.

[5] Y. J. Cho, X. Qin, and J. I. Kang, Convergence theorems based on hybrid methods for generalized equilibrium problems and fixed point problems, Nonlinear Anal. 71 (2009), no. 9, 4203-4214.

[6] P. Cholamjiak, A hybrid iterative scheme for equilibrium problems, variational inequality problems and fixed point problems in Banach spaces, Fixed Point Theory Appl. 2009 (2009), Article ID 719360, 18 pages.

[7] P. Cholamjiak and S. Suantai, A new hybrid algorithm for variational inclusions, generalized equilibrium problems and a finite family of quasi-nonexpansive mappings, Fixed Point Theory Appl. 2009 (2009), Article ID 350979, 20 pages.

[8] W. Cholamjiak and S. Suantai, Monotone hybrid projection algorithms for an infinitely countable family of Lipschitz generalized asymptotically quasi-nonexpansive mappings, Abstr. Appl. Anal. 2009 (2009), Article ID 297565, 16 pages.

[9] P. L. Combettes and S. A. Hirstoaga, Equilibrium programming in Hilbert spaces, J. Nonlinear Convex Anal. 6 (2005), no. 1, 117-136.

[10] A. Genel and J. Lindenstrauss, An example concerning fixed points, Israel J. Math. 22 (1975), no. 1, 81-86.

[11] A. Kangtunyakarn and S. Suantai, Hybrid iterative scheme for generalized equilibrium problems and fixed point problems of finite family of nonexpansive mappings, Nonlinear Anal. Hybrid Syst. 3 (2009), no. 3, 296-309.

[12] T. H. Kim and H. K. Xu, Strong convergence of modified Mann iterations for asymptotically nonexpansive mappings and semigroups, Nonlinear Anal. 64 (2006), no. 5, 1140-1152. 
[13] W. R. Mann, Mean value methods in iteration, Proc. Amer. Math. Soc. 4 (1953), 506510.

[14] G. Marino and H. K. Xu, Weak and strong convergence theorems for strict pseudocontractions in Hilbert spaces, J. Math. Anal. Appl. 329 (2007), no. 1, 336-346.

[15] C. Martinez-Yanes and H. K. Xu, Strong convergence of the CQ method for fixed point iteration processes, Nonlinear Anal. 64 (2006), no. 11, 2400-2411.

[16] K. Nakajo, K. Shimoji, and W. Takahashi, Strong convergence theorems by the hybrid method for families of nonexpansive mappings in Hilbert spaces, Taiwanese J. Math. 10 (2006), no. 2, 339-360.

[17] K. Nakajo, K. Shimoji, and W. Takahashi, On strong convergence by the hybrid method for families of mappings in Hilbert spaces, Nonlinear Anal. 71 (2009), no. 1-2, 112-119.

[18] K. Nakajo and W. Takahashi, Strong convergence theorems for nonexpansive mappings and nonexpansive semigroups, J. Math. Anal. Appl. 279 (2003), no. 2, 372-379.

[19] W. Nilsrakoo and S. Seajung, Weak and strong convergence theorems for countable Lipschitzian mappings and its applications, Nonlinear Anal. 69 (2008), no. 8, 26952708 .

[20] Strong convergence theorems for a countable family of quasi-Lipschitzian mappings and its applications, J. Math. Anal. Appl. 356 (2009), no. 1, 154-167.

[21] J. W. Peng, Y. C. Liou, and J. C. Yao, An iterative algorithm combining viscosity method with parallel method for a generalized equilibrium problem and strict pseudocontractions, Fixed Point Theory Appl. 2009 (2009), Article ID 794178, 21 pages.

[22] S. Reich, Weak convergence theorems for nonexpansive mappings in Banach spaces, J. Math. Anal. Appl. 67 (1979), no. 2, 274-276.

[23] D. R. Sahu, H. K. Xu, and J. C. Yao, Asymptotically strict pseudocontractive mappings in the intermediate sense, Nonlinear Anal. 70 (2009), no. 10, 3502-3511.

[24] N. Shahzad and H. Zegeye, Strong convergence of an implicit iteration process for a finite family of generalized asymptotically quasi-nonexpansive maps, Appl. Math. Comput. 189 (2007), no. 2, 1058-1065.

[25] K. Shimoji and W. Takahashi, Strong convergence to common fixed points of infinite nonexpansive mappings and application, Taiwanese J. Math. 5 (2001), no. 2, 387-404.

[26] A. Tada and W. Takahashi, Weak and strong convergence theorems for a nonexpansive mapping and an equilibrium problem, J. Optim. Theory Appl. 133 (2007), no. 3, 359370.

[27] W. Takahashi, Weak and strong convergence theorems for families of nonexpansive mappings and their applications, Ann. Univ. Mariae Curie-Sklodowska Sect. A 51 (1997), no. 2, 277-292.

[28] W. Takahashi, Y. Takeuchi, and R. Kubota, Strong convergence theorems by hybrid methods for families of nonexpansive mappings in Hilbert spaces, J. Math. Anal. Appl. 341 (2008), no. 1, 276-286.

[29] H. K. Xu, Inequality in Banach spaces with applications, Nonlinear Anal. 16 (1991), no. 12, 1127-1138.

[30] Y. Yao, Y. J. Cho, and Y. Liou, Algorithms of common solutions for variational inclusions, mixed equilibrium problems and fixed point problems, European J. Oper. Res. 212 (2011), no. 2, 242-250.

[31] H. Zhou, Strong convergence theorems for a family of Lipschitz quasi pseudocontractions in Hilbert spaces, Nonlinear Anal. 71 (2009), no. 1-2, 120-125.

[32] H. Zhou and Y. Su, Strong convergence theorems for a family of quasi-asymptotic pseudo-contractions in Hilbert spaces, Nonlinear Anal. 70 (2009), no. 11, 4047-4052. 
Prasit Cholamjiak

School of ScIENCE

University of Phayao

Phayao 56000, Thailand

E-mail address: prasitch2008@yahoo.com

WATCHARAPORN CHOLAMJIAK

SCHOOL OF SCIENCE

University OF PHAYAO

Phayao 56000, Thailand

E-mail address: c-wchp007@hotmail.com

Suthep SuANTAI

Department of Mathematics

FACULTy OF SCIENCE

Chiang Mai University

Chiang Mai 50200, Thailand

E-mail address: scmti005@chiangmai.ac.th 